\title{
Oral immune priming with Bacillus thuringiensis induces a shift in the gene expression of Tribolium castaneum larvae
}

\author{
Jenny M. Greenwood ${ }^{1 \dagger}$, Barbara Milutinović ${ }^{1,2 \dagger}$, Robert Peuß ${ }^{1,3}$, Sarah Behrens ${ }^{1}$, Daniela Esser ${ }^{4}$, Philip Rosenstiel ${ }^{4}$, \\ Hinrich Schulenburg ${ }^{5}$ and Joachim Kurtz ${ }^{1 *}$ (i)
}

\begin{abstract}
Background: The phenomenon of immune priming, i.e. enhanced protection following a secondary exposure to a pathogen, has now been demonstrated in a wide range of invertebrate species. Despite accumulating phenotypic evidence, knowledge of its mechanistic underpinnings is currently very limited. Here we used the system of the red flour beetle, Tribolium castaneum and the insect pathogen Bacillus thuringiensis (Bt) to further our molecular understanding of the oral immune priming phenomenon. We addressed how ingestion of bacterial cues (derived from spore supernatants) of an orally pathogenic and non-pathogenic Bt strain affects gene expression upon later challenge exposure, using a whole-transcriptome sequencing approach.
\end{abstract}

Results: Whereas gene expression of individuals primed with the orally non-pathogenic strain showed minor changes to controls, we found that priming with the pathogenic strain induced regulation of a large set of distinct genes, many of which are known immune candidates. Intriguingly, the immune repertoire activated upon priming and subsequent challenge qualitatively differed from the one mounted upon infection with Bt without previous priming. Moreover, a large subset of priming-specific genes showed an inverse regulation compared to their regulation upon challenge only.

Conclusions: Our data demonstrate that gene expression upon infection is strongly affected by previous immune priming. We hypothesise that this shift in gene expression indicates activation of a more targeted and efficient response towards a previously encountered pathogen, in anticipation of potential secondary encounter.

Keywords: RNA-sequencing, Immune priming, Tribolium castaneum, Host-parasite interaction, Bacillus thuringiensis

\section{Background}

Evolution is a dynamic process and nowhere is this better exemplified than in host-parasite interactions. Hosts must perpetually mount defences in order to ameliorate the damage done by parasites, whilst the parasites themselves must evolve to avoid or temper these defences [1-3]. Such resistance or virulence is achieved by Darwinian processes through selection over several host and parasite generations. However, adaptation to parasites can occur within the individual's lifetime through adaptive immunity,

\footnotetext{
* Correspondence: joachim.kurtz@uni-muenster.de

${ }^{\dagger}$ Equal contributors

'Institute for Evolution and Biodiversity, University of Münster, Hüfferstrasse 1, 48149 Münster, Germany

Full list of author information is available at the end of the article
}

acquired resistance resulting from primary contact that grants survival benefits upon secondary encounter. Acquired immunity was originally thought to be restricted to vertebrates, while invertebrates were supposed to only possess innate immunity. However, evidence from phenotypic analyses have shown that invertebrates may also have some level of immune memory that is often denoted as 'immune priming' [4-8]. A particularly compelling aspect emerging from such studies is that the host response is sometimes specific to the pathogenic agent $[6,9,10]$. Such observations have led to suggestions that the boundary between innate and acquired immunity is blurred [11]. Priming in insects can be achieved by haemocoelic infection (pricking) with bacterial components, inactivated or 
low-dose pathogens $[5,9,12]$ and by oral consumption of live bacteria or bacteria-derived components [13-15]. Despite accumulating evidence for immune priming in insects, knowledge of its mechanistic underpinnings is currently limited (for review see, [16]). Insects possess no known comparable system to vertebrates in terms of an underlying genetic basis for acquired immunity, although some candidates have been proposed, e.g. Dscam [17]. Previously, strategies for finding resistance genes involved a time- and knowledge-intensive candidate gene approach, but with the recent advances in sequencing technologies it has become tractable to efficiently explore insect immunity on a genome-wide basis $[18,19]$. To date, such genomic approaches have rarely been applied to explore the mechanistic basis of immune priming [20,21]. Here, we used the red flour beetle, Tribolium castaneum and the insect pathogen Bacillus thuringiensis to explore the genetic underpinnings of oral immune priming [15]. T. castaneum is a major pest of food grain [22]. This species has become a powerful model organism also for studies of insect immunity, with a fully-sequenced genome [23] and more recently, established protocols for studying host-parasite interactions using the pathogen $B$. thuringiensis $[9,24,25]$. It has previously been shown that $T$. castaneum has enhanced survival to $B$. thuringiensis infection after prior exposure to heat-killed bacteria by pricking infection [9] and after exposure to spore supernatant via the oral route [15]. Gene expression strongly differs for infection with live bacteria for these routes [19]. To further our understanding of insect acquired immunity, we here focussed on the oral route of infection and assessed how the priming treatment affects gene expression upon later challenge exposure. For priming, we used filter-sterilised spore supernatants that do not contain any live bacteria or spores to exclude potentially confounding effects of infection upon priming. In addition, we compared priming responses to two strains of $B$. thuringiensis; one of these strains is orally pathogenic to $T$. castaneum and has previously been identified as an effective priming agent, while the other one is neither pathogenic to $T$. castaneum, nor does priming with it lead to enhanced survival [15]. We compared transcriptomes of primed and non-primed larvae upon challenge and identified a priming-specific pattern of gene expression that was clearly distinct from the infection response. Further analysis revealed a number of candidate genes, which provide a new basis to study the molecular underpinnings of immune priming in insects.

\section{Results}

Priming with Btt elicits a differential gene expression response in $\mathrm{T}$. castaneum

We performed RNA-seq experiments to identify the molecular basis of oral immune priming in T. castaneum larvae [15]. For this, larvae were orally primed with spore-culture supernatants of either the priming-inducing $B t t$ strain, or the Bt407 strain, which does not confer survival benefits upon subsequent infection with $B t t$. Both groups ('Btt' and 'Bt407' priming) and an additional medium-control group ('control' priming) were subsequently orally challenged with Btt spores or were left naïve, resulting in six treatment groups, all in triplicates, i.e. 18 sequenced libraries (see Methods and Fig. 1). On average, 51.6 million raw reads were generated per sample and 46 million reads per sample remained after filtering, of which $78 \%$ could be mapped to the T. castaneum genome. 12288 of the 12777 annotated genes were detected. We found a total of 1610 genes up-regulated and 1448 down-regulated compared to control treatment samples (i.e. control priming with medium and left naïve for challenge). All differentially expressed genes in the different priming-challenge treatments (compared to fully naive control) are listed in Additional file 1: Table S1.

A principle components analysis (PCA, Fig. 2) showed that the primary axis (describing 59\% of the variance) distinguishes among those samples exposed to Btt at any stage in the experiment (priming or challenge, i.e. Btt$B t t, B t t$-Naive, Control-Btt, Bt407-Btt) and those having no contact with $B t t$ at any stage in the protocol (Control-Naive and Bt407-Naive) and therefore separates beetles by expression response to pathogenicity. The secondary axis (17.6\%) distinguishes between beetles that were not primed but were challenged with Btt (Bt407-Btt and Control-Btt), and beetles that were not challenged with Btt (Bt407 -Naive, Control-Naive and Btt-Naive). Btt$B t t$ treated beetles cluster with the latter group, rather than those that were not primed but were challenged with $B t t$. This suggests a profound influence of priming with Btt spore supernatants on beetles challenged with Btt spores.

\section{Oral priming with Btt spore supernatant prepares larvae for subsequent challenge}

Consistent with the PCA, Venn diagrams of differentially expressed genes showed many shared changes in expression for different treatments compared with naive beetles (Fig. 3, Additional file 2: Figure S1). For example, 292 genes were up-regulated exclusively among larvae challenged with Btt (Control-Btt, Bt407-Btt and Btt-Btt) and 307 were shared exclusively among $B t t$-challenged larvae that had not been primed or were primed with Bt407 (Control-Btt and Bt407-Btt), confirming a strong transcriptional response to $B t t$ spore ingestion [19]. Interestingly, 315 genes were specifically up-regulated only in Btt-primed larvae (Btt-Naive, Btt-Btt), showing that $B t t$-priming drastically altered gene expression patterns. Large differences in gene regulation were generated in larvae primed by the two $B t$ strains. Few genes in total 


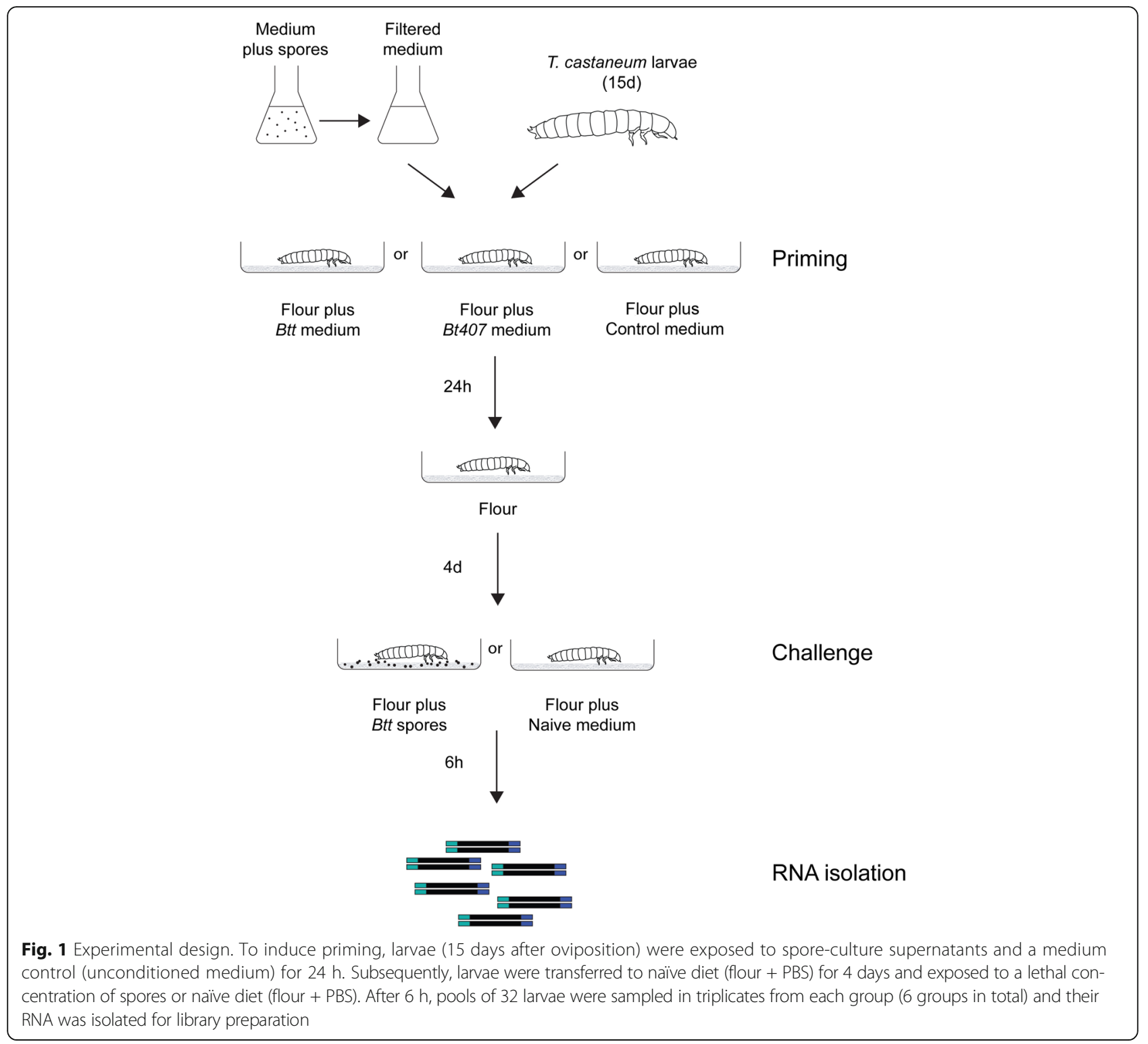

(61) were up-regulated in larvae primed with Bt407 (Bt407-Naive) compared with control (Control-Naïve) treatment, consistent with phenotypic data that shows that Bt407 confers no priming advantage [15]. However, 112 genes were exclusively down-regulated in Bt407Naive larvae, suggesting that larvae do respond to Bt407 cues in their diet, but that any changes in gene regulation do not confer protection upon challenge. Furthermore, since large numbers of differentially expressed genes were exclusively shared in both Btt-primed groups (Btt-Naive, $B t t-B t t)$, our results indicate that crucial changes leading to the priming effect already occur before the challenge (during the 4 days between priming and RNA sampling, Fig. 1), strongly influencing gene expression pattern upon challenge itself $(B t t-B t t)$.

\section{Immune pathways}

To further analyse the effect of priming and challenge on immune gene expression, we tested whether certain categories of immune genes identified in T. castaneum by Zou et al. [26] showed an enrichment for up- or down-regulated genes (Fig. 4). To investigate in detail the potential role of the Toll and IMD pathways, we also focussed on key components of these pathways and compared their expression between the treatments, as described in Behrens et al. [19] (Fig. 5).

We found a strong signal of differential expression of immune genes (Fig. 4), such that immune genes were overrepresented among both up- and down-regulated genes in all treatment groups, except for Bt407-primed and unchallenged beetles, which also did not show any 


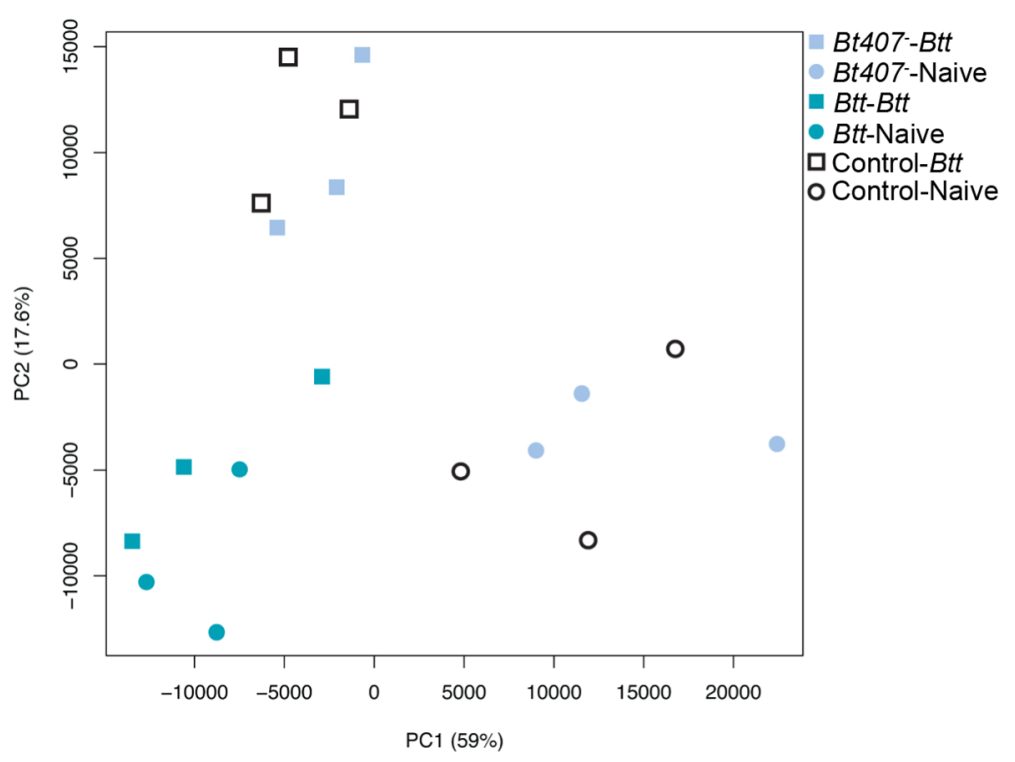

Fig. 2 Principle component analysis for all samples $6 \mathrm{~h}$ after exposure to Btt-contaminated or naive diet. The plot shows clustering of samples according to treatment group and replicate, based on FPKM expression values and the percentage of variance described by the first two principle components

significant enrichment for up-regulated genes in any of the immune subcategory. All other treatment groups showed upregulation of Toll pathway components and extracellular signalling categories. Antimicrobial peptide genes (AMP) genes and genes involved in cellular responses were only up-regulated upon Btt challenge, regardless of priming condition. In contrast, recognition genes and reactive oxygen species (ROS) genes were already up-regulated upon priming with Btt (but not Bt407), even when this was not followed by infection, suggesting that priming leads to an increased 'alertness' by up-regulation of immune recognition genes and an elevated level of reactive oxygen based defences. It is further noteworthy that IMD genes that were up-regulated upon infection without priming (Control-Btt and Bt407-Btt) did not show differential regulation when there was previous priming with Btt. Finally, in the Bt407 -Naive treatment groups, the ROS, AMP and Toll pathways were significantly down-regulated compared with control-primed beetles.

Upon closer inspection of the Toll and IMD pathways, we found many differences for the Btt challenged larvae in relation to whether they had been primed with $B t t$ or Bt407 (Fig. 5). In detail, several genes of the classical immune pathways (e.g. PGRP-LA, Dif, Basket, IAP, Kenny) were up-regulated upon challenge following inefficient priming (with Bt407), suggesting an overall activation of these pathways. In contrast, we found only a reduced number of key genes of the Toll and IMD pathway to be activated in the $B t t$ priming-Btt challenge scenario.
Genes with known functions against B. thuringiensis and other pathogens are up-regulated upon priming with $\mathrm{Btt}$ To narrow down candidates among the genes differentially regulated upon priming (groups Btt-Naive and Btt-Btt; Fig. 3), we screened the literature for known pathogenrelated functions of those genes. We found several candidates with a described role in insect immunity to be differentially regulated (Fig. 6, Additional file 3: Figure S2, Additional file 4: Table S2). For example, lysozyme (TC010351) and many of the c-type lectin genes (e.g. TC003708, TC010419) were strongly up-regulated, and have a known function in defence against a variety of bacteria. Lysozymes cut bacterial cell wall components [2729 ] and c-type lectins play an important role in pathogen recognition and opsonisation [30-32], and were recently proposed to contribute to specific immune responses, especially in invertebrates [33]. Furthermore, two phospholipase A2 genes (TC015181, TC005550) were found upregulated upon priming (Fig. 6, Additional file 3: Figure S2). These enzymes participate in the formation of eicosanoids from arachidonic acid [34, 35] and were found to play multiple immune roles in insects, such as in nodulation, prophenoloxidase activation [36, 37] and phagocytosis [34], including responses to bacterial challenge and Toll and Imd pathway activation in T. castaneum [35].

It is further noteworthy that we found several genes with a known defence function against orally ingested pathogens and specifically, against $B$. thuringiensis, exclusively in the Btt primed larvae (Btt-Naïve, Btt-Btt). Particularly interesting is the hexamerin gene (TC005375), 


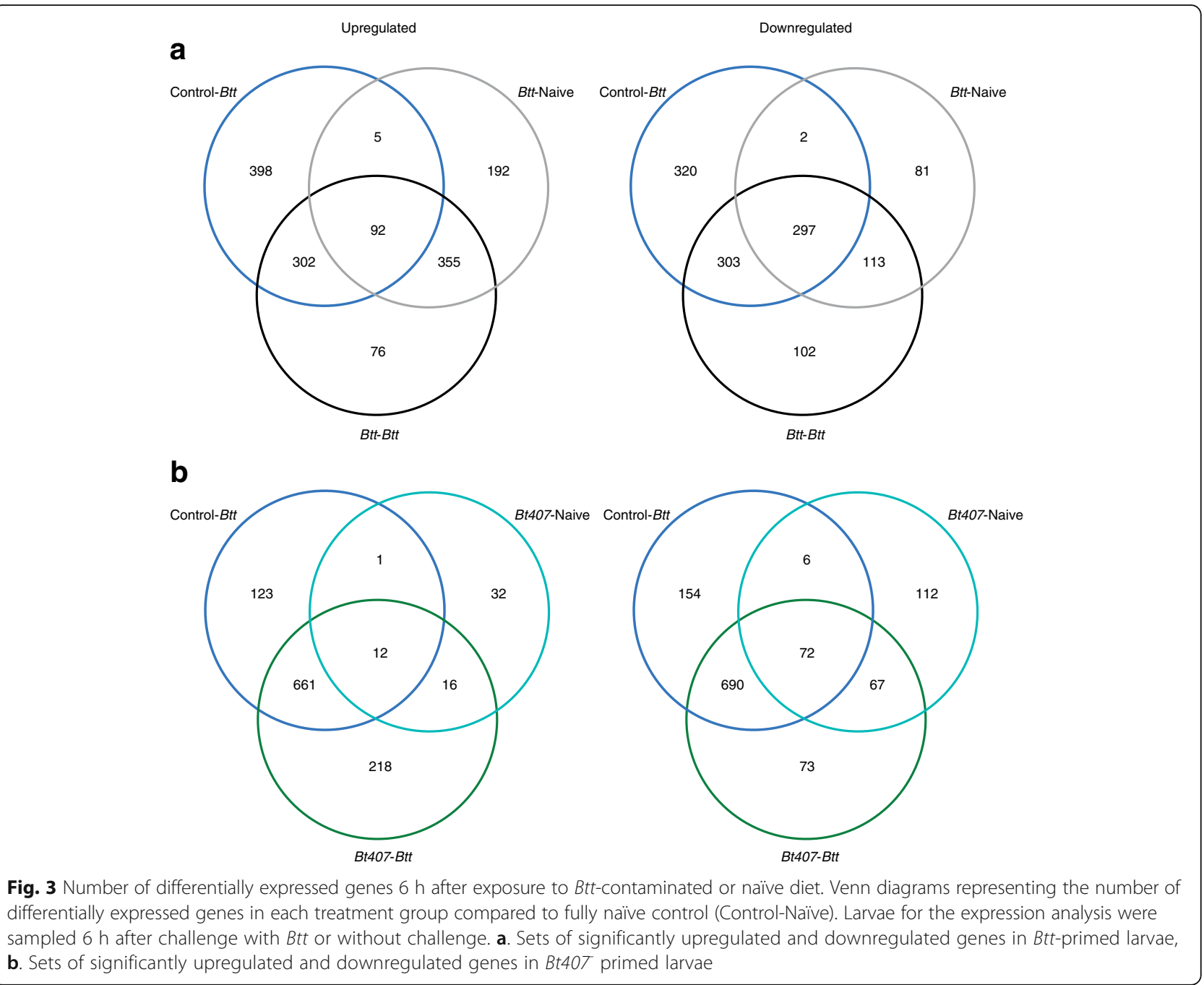

which we found strongly up-regulated compared to control (Fig. 6, Additional file 3: Figure S2). Hexamerin expression and protein concentration were found increased after a bacterial challenge [38-41]. In Drosophila, hexamerin was indicated to function as a humoral pro-coagulant, participating in bacteria binding and clot formation [42]. Interestingly, increased hemolymph and gut hexamerin concentrations were found to play a role in the reaction of the cotton bollworm to Cry1Ac toxins produced by a $B$. thuringiensis strain, by aggregating with the toxin crystal and forming an insoluble coagulant [43-45]. A putative Hdd1 defense protein gene (TC013059), associated with gut immune defence and resistance to $B$. thuringiensis toxins was found strongly expressed upon Btt priming (Fig. 6, Additional file 3: Figure S2). The expression of this gene was upregulated after ingestion of bacteria-contaminated diet in the cabbage semilooper [38] and a similar gene was found to be involved in prophenoloxidase activation and nodulation response in the cotton bollworm [46]. Interestingly, a gene of the same class (Hdd11) was found induced in the Cry $1 \mathrm{Ab}$ resistant sugarcane borer [47] and in the beet armyworm after ingestion of $B$. thuringiensis VIP toxins [48], indicating its importance in defence against $B$. thuringiensis.

\section{Immune priming differs from challenge}

We found that $9 \%$ (78 of the total 825) of the genes upregulated upon challenge with $B t t$ after previous priming $(B t t-B t t)$ were in fact downregulated in beetles challenged without priming (Control-Btt). The different signature of priming $v s$. challenge was seen in the overrepresentation of gene ontology (GO) terms in the different treatments (Fig. 7, Additional file 5: Table S3). "Structural constituent of cuticle" was the most strongly overrepresented term among up-regulated genes when the larvae were primed with Btt (Btt-Naive and Btt-Btt), whereas in larvae only 


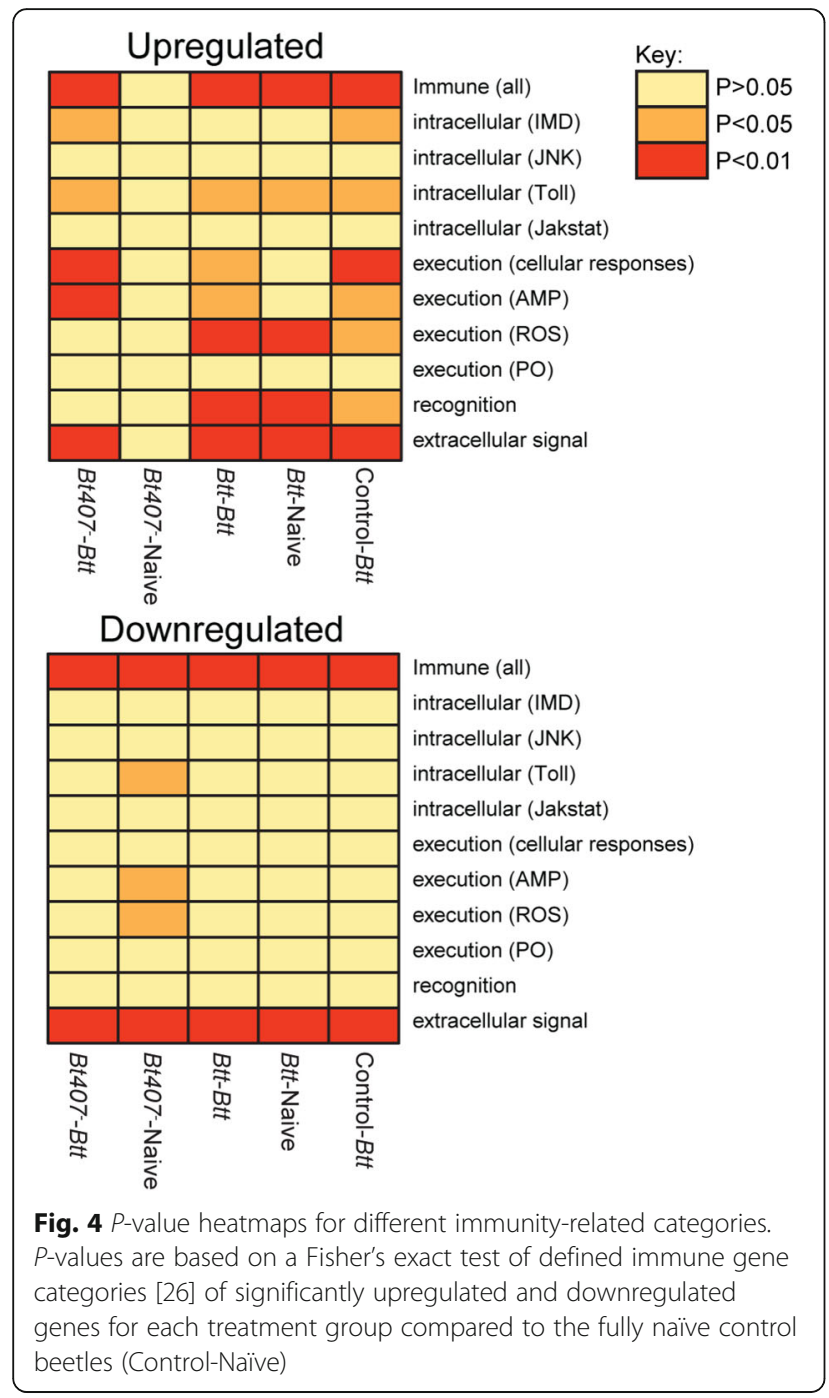

challenged with Btt (Control-Btt), "serine-type endopeptidase activity" was the most significantly up-regulated term. Interestingly, this GO term was most significantly down-regulated in larvae only primed with Btt (Btt-Naïve), suggesting an inverse pattern of gene regulation in primed, compared to challenged-only larvae.

When we looked at only Btt primed individuals that were not challenged with live spores (Btt-Naïve), we found several immune candidates downregulated in this treatment, but upregulated upon challenge-only (Fig. 6, Additional file 3: Figure S2). These were the two genes annotated as variable lymphocyte receptors (TC004538, TC004541), a chemosensory protein Csp 18 (TC008674) as well as two serine peptidase inhibitors (TC005750, TC015224) and a spätzle (TC000520). Conversely, a pathogenesis related protein (TC000516), odorant receptor 150 (TC030347), and several Osiris genes (TC012679, TC011827, TC011661, TC011660, TC011658, TC012820, TC012821) were found up- regulated upon priming, but down-regulated upon challenge (Fig. 6, Additional file 3: Figure S2). Even though different time-points after priming (4 days) and challenge (6h) are here considered, these observations, together with the data shown above, strongly indicate that immune priming differs from challenge.

\section{Discussion}

In this study, we aimed at exploring the molecular underpinnings of oral immune priming in $T$. castaneum larvae [15] using a whole-animal transcriptome sequencing approach. We analysed host gene expression profiles after exposure to bacterial cues (culture supernatants) of two different strains; an orally pathogenic strain $(B t t)$ inducing a priming response and conferring survival benefits upon challenge, and a non-pathogenic strain (Bt407) where this effect remains absent. Consistent with phenotypic results [15], we found strong differences in host gene expression profiles between the two bacterial strains. Whereas priming with $B t 407$ showed only minor differences compared to the control-priming group in terms of up-regulated genes, priming with $B t t$ induced a large set of differentially regulated genes (Figs. 3 and 6, Additional file 3: Figure S2), indicating strong responses of the host to the $B t t$ priming diet. Note that priming in our study is achieved by using sterilised spore culture supernatants, such that observed gene expression changes cannot be attributed to an active infection in the host.

We expected two hypothetical scenarios by which immune priming could take place $[16,49]$. In the first, priming could induce a sustained immune response that would prophylactically confer a survival benefit upon secondary challenge with live $B t t$ [5, 50]. Indeed, we found support for this scenario in our dataset, i.e. gene expression changes upon priming that remained active until the challenge. For example, several genes of the Toll and IMD pathways were found activated prior challenge (i.e. also in the Btt_Naive group; Fig. 5, right upper panel), which could represent an anticipatory response based on potentially higher amounts of antimicrobial peptides that are 'ready-to-use' upon challenge. This hypothesis is also based on the fact that the Toll and IMD pathways are in a somewhat 'active state' (i.e. upregulation of upstream factors such as PGRP-LE for IMD and Spätzle for Toll) after priming with Btt, when not followed by challenge. Second, priming could lead to an anticipatory immune defence that is recalled upon challenge, such that a stronger and/or faster response could take place, facilitating the rapid elimination of pathogens. Such priming would rely on immune memory in a more narrow sense, i.e., the ability of an immune system to store and recall the information on a previously encountered pathogen $[7,8,51]$. In both of the above mentioned cases (sustained or recalled), the type of response 


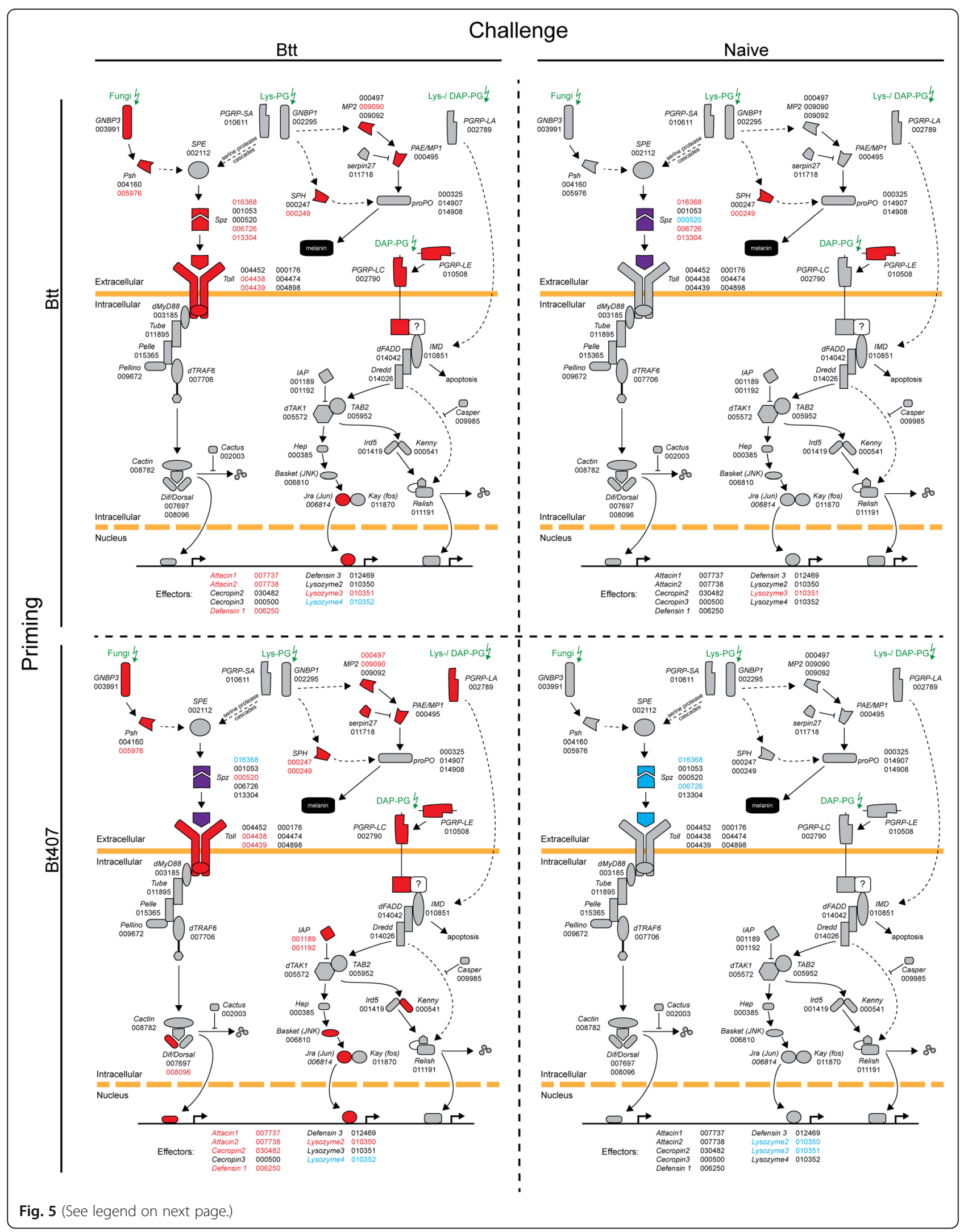


(See figure on previous page.)

Fig. 5 Regulation of the Toll and IMD pathway in relation to priming and challenge treatments. Illustrated are the Toll and IMD pathways after Zou et al., [26] for the two priming treatments, $6 \mathrm{~h}$ after the challenge with Btt spore-contaminated or naïve diet (flour + PBS). Red color indicates significant upregulation, blue significant downregulation of the respective genes and purple both, significant up- and downregulated genes in the case when genes from the same gene family were reversely expressed. The corresponding official gene IDs ('TC\#\#\#\#\#') are specified next to the genes. For effectors, only those that are differentially expressed $6 \mathrm{~h}$ after the challenge treatment (Btt or naïve) in at least one treatment are indicated with their respective official gene IDs

upon priming and challenge would be qualitatively similar. Such a scenario was recently observed in transgenerational immune priming in bumble bees, where worker offspring coming from primed queens exhibited overlapping expression signatures with workers that were directly exposed, but whose mothers were not primed [20].

By contrast, the activated immune repertoire that we here observed upon priming seems to a certain degree to be qualitatively different from the one mounted upon infection without a previous priming. Intriguingly, a similar observation of a qualitatively distinct priming response was recently reported for immune priming in the snail Biomphalaria glabrata with the trematode parasite Schistosoma mansoni $[21,52]$. In this system, priming resulted in a shift from cellular to humoral immune reactions. When comparing groups that were challenged with or without preceding priming in our study, we found a large set of genes regulated in the Btt primed$B t t$ challenged group, many of which are known immune candidates. Since the majority of those genes were already regulated in the group receiving no challenge (Btt-Naïve), this suggests that the observed survival benefit in $T$. castaneum larvae results from the induction of a specific set of genes, early after the exposure to bacterial cues in their environment, and that such induction results in largely different gene expression than the one activated upon challenge without the previous priming. It would be interesting to see how prevalent these scenarios are among invertebrate phyla, i.e., whether the priming gene expression is different or similar compared to a secondary challenge.

Compared to Drosophila, T. castaneum seems to mount a rather promiscuous immune response after bacterial challenge, by concomitantly upregulating Toll and IMD pathways in response to bacteria $\left(\mathrm{Gram}^{-}\right.$or

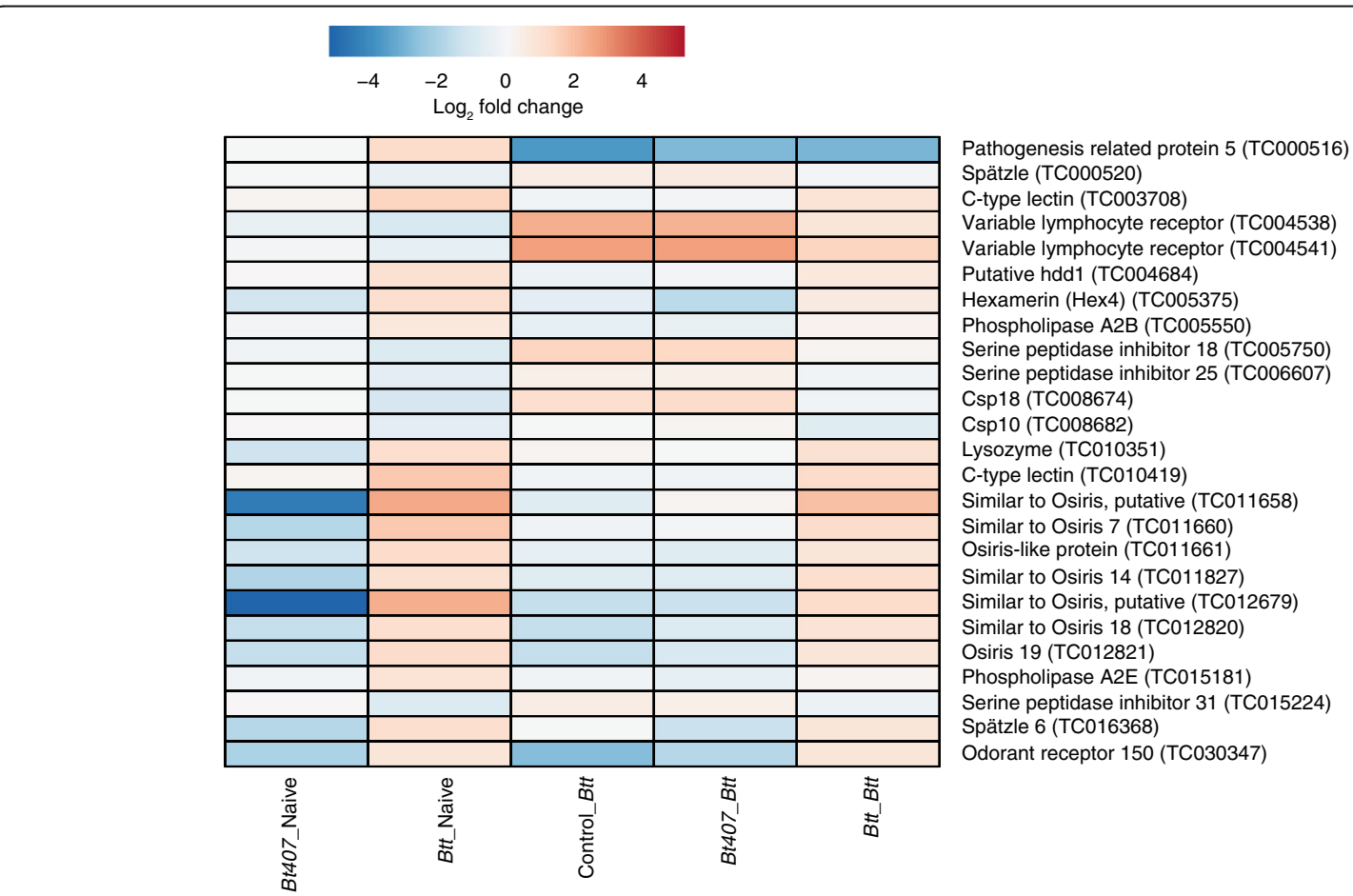

Fig. 6 Heatmap of differentially regulated candidate immunity-related genes. Heat maps of a subset of candidate immunity-related genes regulated upon priming or showing reversed expression compared to groups challenged without or after ineffective priming. Heat maps are based on the log2 fold change expression values. Genes are sorted according to increasing TC numbers. Please note that gene descriptions for T. castaneum often come from automatic annotations and are not always verified by functional analyses 


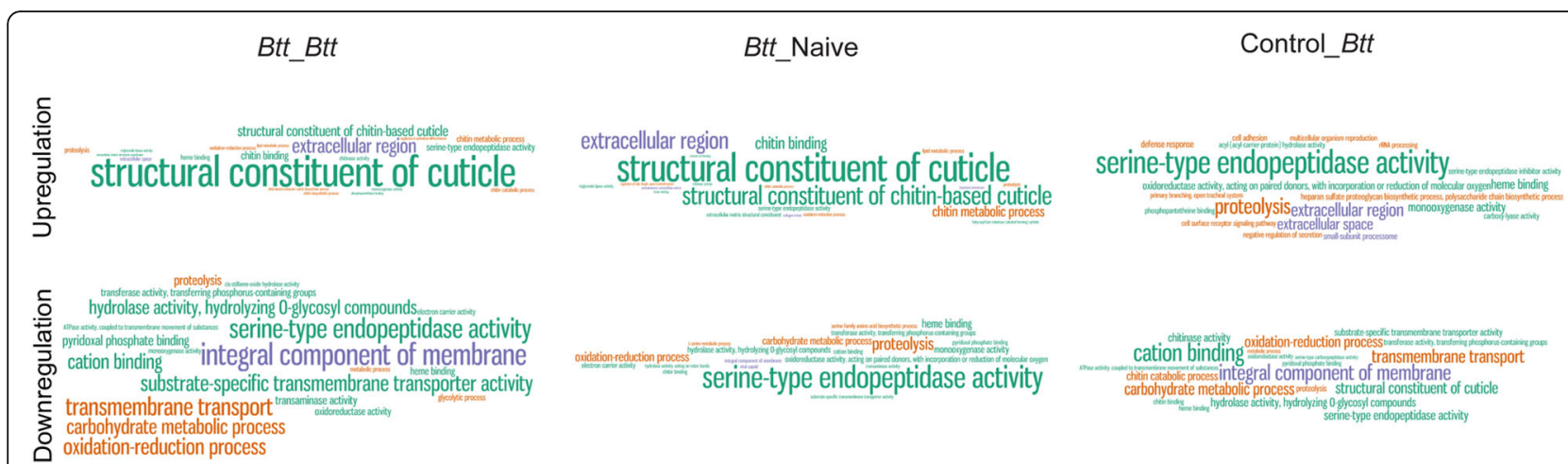

Fig. 7 Word clouds of overrepresented GO terms in sets of differentially expressed genes. The R package TopGO was used to perform GO enrichment analyses for each set of differentially expressed genes in different treatment groups, using the weight01 GO graph algorithm and a Fishers Exact Test for significance [74]. P-values were scaled according to the scaling factor - $\log _{10}(p$-value) and the top 20 terms were visualised in Wordle ${ }^{\text {TM }}$

$\mathrm{Gram}^{+}$) $[53,54]$. Indeed, challenge without the previous priming induced an overall regulation of the Toll and IMD pathways in our study as well (Figs. 4 and 5, left lower panel); however, only a reduced number of those genes were upregulated upon challenge when there was previous priming (Figs. 4 and 5, left upper panel). We even found many of the priming-specific genes to show an inverse regulation compared to their regulation upon challenge without the priming, a pattern that was also visible in the different GO term overrepresentation results (Fig. 7). We hypothesise that this "shift" in gene expression indicates activation of a more targeted and more efficient response following information on previously encountered pathogens, in anticipation of a potential secondary encounter. For example, priming induced several novel effector genes, not normally expressed upon infection, like hexamerin, pathogenesis related protein 5 , lysozyme and hdd1 defence protein, all with a reported role in defence against orally ingested pathogens and $\mathrm{Ba}$ cillus thuringiensis. Activation of a narrower, more suitable response type towards a specific pathogen would certainly prove beneficial when an infection is expected.

It is difficult to narrow down the oral priming response to only a few candidate genes. Rather, priming with $B t t$ seems to mount an orchestrated response of various signalling and effector molecules, together contributing to survival benefits upon secondary exposure to lethal concentration of spores. Of note is however, that in order to understand phenotypic data, we looked at gene regulation 4 days after priming had occurred. Although this still had a strong influence on gene expression upon challenge (Btt_Btt treatment group, Figs. 3 and 6, Additional file 3: Figure S2), it may be that looking at earlier timepoints would reveal important candidates more clearly (e.g., by stronger regulation). Hexamerin though, could play a particularly important role. Hexamerins, or similar lipoproteins were found to coagulate and bind $B t$ toxins, rendering them inactive $[44,45]$. Strong hexamerin regulation may suggest formation of a trap by crosslinking with other hexamerin molecules, thus facilitating binding of the toxins and/or bacteria and inactivating them $[44,45]$. Hexamerins may therefore serve as a first line of defence in the gut [45], especially if accumulating in the gut upon priming, trapping the toxins and bacteria and preventing them from reaching the midgut epithelium.

Of note here is that the information on gene identity in T. castaneum often comes from automatic annotations based on sequence similarities and while some genes are well annotated (e.g., hexamerin, lysozyme), others (e.g., variable lymphocyte receptors, Fig. 6) are not verified by functional analyses. Similarly, we found many genes strongly regulated for which no annotation so far exists or with no described function in other insects, as is the case for the many Osiris-like genes with characteristic expression patterns (Fig. 6). The Osiris gene family is well-conserved across insects, but its function is so far largely unknown $[55,56]$.

It is not clear which bacteria-derived components induce priming in our system, as the supernatant of a sporulating culture may be abundant in various candidate molecules or pathogen-associated molecular patterns; remnants of the cell wall components remaining after the cell lysis or secreted non-proteinaceous components or toxins that remained in the supernatant after filter-sterilisation. Such bacterial cues might damage the host tissue and serve as a danger signal, activating host immunity [57], which could be absent in the non-pathogenic Bt407 strain. In this context, it is noteworthy that Bt407, in contrast to Btt, does not have the beetle-specific Cry toxin that breaks the gut barrier. However, upon preparation of the priming diet, bacterial culture is centrifuged such that no crystal toxins should remain in the supernatnats, except potentially in a form of loose monomers, but this needs to be investigated. Alternatively, certain molecules might be affecting the 
resident microbiota, further promoting a primed response. Indeed, a recent study showed that, similar to the mosquito-Plasmodium system [14, 58], microbiota play a crucial role in the oral priming in our system as well [59], but the mechanisms for this dependence and how they relate to the specific gene expression patterns found here are still unknown. Interestingly, we found PGRP-LE activated after priming with Btt (Btt_Naive, Fig. 5, right upper panel), which is a key gut bacterial sensor in Drosophila involved in balanced responses to pathogenic bacteria and microbiota [60]. Further research is needed to see whether and how this interesting gene is involved in microbiota regulation of priming in our system.

Regarding the question of specificity of priming responses, it is interesting that the 'non-efficient' Bt407 priming was not without any effects. Compared to fully naive controls, we found a quite clear pattern of downregulation of genes (Fig. 3), in particular for Toll, AMP and ROS-mediated defences (Fig. 4), which might also be the cause for the observed absence of enrichment for up-regulated immune recognition upon Btt challenge (Fig. 4). However, the reasons for these effects are currently unclear and need further study.

A puzzling question is, why a potentially more effective immune response would be activated only when priming occurred, but not constitutively or upon challenge without priming? First, as with other immune defence, priming with Btt seems to be costly; primed larvae grow and develop more slowly than the controls [15]. It thus may depend on the epidemiology as to whether it is worth paying these costs. Second, in periods of high bacterial load in a population of beetles, priming might actually regularly occur, e.g. via cannibalising infected larvae [61]. Priming-eliciting cues could be present in cadavers as the result of bacterial sporulation such that eating infected cadavers could prove beneficial for survival. However, little is currently known about $B t$ epidemiology and how host and pathogen interact in nature; these topics require further research.

\section{Conclusions}

We here show that oral priming with spore culture supernatants of $B$. thuringiensis tenebrionis is achieved by extensive transcriptome changes in $T$. castaneum that are specific to priming with $B t t$, but absent from priming with the non-infectious strain Bt407. A unique pattern of gene expression was found that is different from challenge without the previous priming. Such a shift in the expression pattern towards a potentially more effective response is very intriguing and it would be interesting to test if this phenomenon is bacteria-specific and whether such a response type could also be found in other invertebrates that show immune priming.

\begin{abstract}
Methods
Insects

For all experiments we used the wild type strain of Tribolium castaneum, Croatia 1 (Cro1), which was collected in May 2010 in Croatia [24]. This strain was adapted to lab conditions for more than 20 generations ( 18 months). Beetles were reared on flour (type 550 ) with $5 \%$ brewer's yeast at $30^{\circ} \mathrm{C}$ with a $12 / 12 \mathrm{~h}$ light/dark cycle and $60 \%$ relative humidity.
\end{abstract}

\section{Bacteria and spore cultivation}

Bacillus thuringiensis bv. tenebrionis (Btt) was obtained from the Bacillus Genetic Stock Center (BGSC, Ohio State University, USA) and Bacillus thuringiensis 407 (Bt407) was kindly provided by Dr. Christina NielsenLeroux, Institut National de Recherche Agronomique (La Minière, 78285 Guyancourt Cedex, France). Before using in experiments, bacteria were subcloned five times on LB-Agar and glycerin stocks were stored at $-80^{\circ} \mathrm{C}$. Spore cultures of Btt and Bt407- were produced as previously described [24] and centrifuged at $2900 \mathrm{x} g$ at room temperature for $12 \mathrm{~min}$. Spores were washed and subsequently resuspended in phosphate buffered saline (PBS, Calbiochem ${ }^{\circ}$ ), counted using a Thoma counting chamber $(0.02 \mathrm{~mm}$ depth$)$ and used for challenge immediately.

\section{Experimental design}

The priming experiment was done as in Milutinović et al. [15] (see Fig. 1). For this, spore cultures of Btt and Bt $407^{-}$were centrifuged and the supernatants subsequently filter-sterilised, first using a $0.45 \mu \mathrm{m}$ and then a $0.2 \mu \mathrm{m}$ cellulose acetate filter (Whatman $\mathrm{GmbH}$ ). Flour with yeast was added to the supernatant $(0.15 \mathrm{~g} / \mathrm{mL}$ of supernatant). Diet for the control larvae was prepared by mixing the flour with sterile sporulation media. Such liquid diet was pipetted into wells of a 96-well plate (Sarstedt, Germany) and dried in the oven at $36^{\circ} \mathrm{C}$ overnight. The next day, similar-sized $T$. castaneum larvae (15 days after a 24 h oviposition) that were cultivated under standard conditions were individually exposed to the priming or control diet (sterile sporulation media) for $24 \mathrm{~h}$ and transferred to a naive diet of flour discs obtained by mixing flour and PBS. Larvae were kept on the naive diet for 4 days after which they were similarly exposed to spore-containing $\left(5 \times 10^{9} \mathrm{~mL}^{-1}\right.$ Btt spores in PBS mixed with flour) or naive diet for $6 \mathrm{~h}$ and sampled for the transcriptome analysis. This timepoint was used since our previous study showed that sampling $6 \mathrm{~h}$ after the challenge gives a clear expression signature, compared to already weaker expression after $18 \mathrm{~h}$ [19]. Hence, the sampled treatments were as follows: $B t t$ primed-Btt challenged (Btt-Btt), Bt407 primed-Btt challenged (Bt407-Btt), Control-Btt challenged (Control-Btt), Btt primed-Naïve 
(Btt-Naive), Bt407 primed-Naïve (Bt407-Naïve), ControlNaïve. Each treatment was replicated 3 times, with a pool of 32 larvae each.

\section{Sample preparation, library construction and sequencing} For each treatment group, three replicate RNA libraries, each consisting of the 32 pooled T. castaneum individuals were produced. Total RNA from frozen beetles was isolated using mirVana ${ }^{\mathrm{TM}}$ miRNA Isolation Kit (Ambion) according to the instructions of the manufacturer. The libraries for the whole transcriptome sequencing were created with the Illumina TruSeq RNA Library Prep kit (version February 2012, Part\# 15026495 Rev. B). After cluster generation on the cBot with the TruSeq PE Cluster Kit v3, the sequencing was performed with the TruSeq SBS Kit v3 (200 cycles) on two lanes of the Illumina HiSeq 2000.

\section{Transcriptomic analysis}

The transcriptomic assembly and analysis closely followed the procedures described by Behrens et al. [19]. Before mapping, a number of filtering steps were performed on the data. Firstly, Illumina quality-failed reads were removed from the read files, and adaptor sequences were removed using the package SeqPrep [62]. Then Seqtk [63] was used to trim the first 13 base pairs of sequence from the reads to remove biases in nucleotide composition due to random hexamer priming [64], which improved the number of reads mapping to the genome.

After filtering, Tophat v2.0.11 [65] was used to map the reads to the Tribolium 3.0 reference genome downloaded from Beetlebase (Kim et al. [66]). A separate, more recent annotation file, incorporating improved gene models taking advantage of transcriptomic data was downloaded from the iBeetle website $[67,68]$ and used to guide the mapping process, as well as the subsequent assembly and differential expression analyses.

Next, Cufflinks v.2.2.1 [69] was used to quantify the transcripts against the reference.gtf file, using default parameters. Cuffmerge was used to merge the individual assemblies into a comprehensive transcriptome and the Cuffdiff utility [70] was used to normalise the data using upper-quartile normalisation and to quantify differential expression of genes across samples; a value of $p<0.05$, FDR $<0.05$ was used to identify genes with significant differential expression. Data were imported into R [71] for further statistical analysis.

Principle components analysis (PCA) was used to summarise the distribution of gene expression values of the samples using functions in the $\mathrm{R}$ base package and Venn diagrams were generated for the data using the $R$ package VennDiagram [72].

In order to generate functional terms associated with the genes of interest, the software Blast2GO [73] was used to annotate the iBeetle T. castaneum genome annotation [67]. The $\mathrm{R}$ package TopGO was then used to generate GO enrichments for each of our treatment comparisons of interest, using the weight01 GO graph algorithm and Fishers exact test for significance [74]. Results of the 20 most significant terms were visualised with Wordle ${ }^{\mathrm{Tx}}$ after the $p$-values were scaled according to $-\log _{10}(p$-value) $[19,75]$. Subsequently, these results were merged with Gene Ontology terms [76] downloaded from Ensembl Biomart [77] and results of a BlastP search [78] using default parameters, to further add gene information for interpretation of the results.

Finally, immunity genes identified by Zou et al. [26] were tested for enrichment in up- or downregulated genes of each treatment using a Fisher's exact test. $P$ values were normalised using the Benjamini-Hochberg correction method [79]. Zou et al. [26] identified around 300 candidate defense proteins based on sequence similarity to homologs known to participate in immune responses. They further characterized these genes with phylogenetic analyses of immune gene families and RTqPCR analyses after bacterial and fungal pricking.

\section{Additional files}

\begin{abstract}
Additional file 1: Table S1. Lists of all differentially expressed genes in the different priming-challenge treatments (compared to the fully naïve control; xlsx file). Relevant columns include the following: sample_1 and sample_2 - treatment groups being compared; Normalised FPKM sample_1 and sample_2 - FPKM of samples being compared; $\log 2$ (fold_change) - log2(FPKM sample 2/FPKM sample 1), i.e. negative means sample 1 upregulated compared with sample 2, positive means sample 2 upregulated compared with sample 1; cuffdiff test_statistic - test statistic of differential expression test; $p \_$value $-p$-value of differential expression test; q_value (FDR correction) - adjusted $P$-value of differential expression test. (XLSX $598 \mathrm{~kb}$ )
\end{abstract}

Additional file 2: Figure S1. Number of differentially expressed genes $6 \mathrm{~h}$ after exposure to Btt-contaminated or naïve diet. Venn diagrams representing the number of differentially expressed genes in each treatment group compared to fully naïve control (Control-Naïve). Larvae for the expression analysis were sampled $6 \mathrm{~h}$ after challenge with Btt or without challenge. A. Sets of significantly upregulated genes in all treatments, B. Significantly downregulated genes in all treatments. (PDF $1.12 \mathrm{~kb}$ )

Additional file 3: Figure S2. Barplots of candidate immunity-related genes. Barplots of a subset of candidate immunity-related genes regulated upon priming or showing reversed expression compared to groups challenged without or after ineffective Bt407 priming (see also Fig. 6.) Y-axis shows FPKM values. Error bars show 95\% confidence intervals. Please note that gene descriptions for $T$. castaneum often come from automatic annotations and are not always verified by functional analyses. (PDF $9.42 \mathrm{~kb}$ )

Additional file 4: Table S2. Gene description summary for Additional file 3: Figure S2. The table shows the au2 and TC numbers as well as the gene description for the genes shown in Fig. 6 and Additional file 3: Figure S2. Please note that gene descriptions for $T$. castaneum often come from automatic annotations and are not always verified by functional analyses. (DOCX $69 \mathrm{~kb}$ )

Additional file 5: Table S3. Lists of top 20 overrepresented GO terms in Btt exposed treatment groups (compared to fully naïve control). Methodology as described for Fig. 7. The columns are as follows: GO ID - Gene Ontology unique ID of the overrepresented GO term; GO term - text descriptor of the overrepresented GO term; total no.genes 
annotated - total number of genes in the $T$. castaneum genome which are annotated with this GO term; observed - number of genes over- or underrepresented in the treatment found to be annotated with this GO term; expected - number of genes over- or underrepresented in the treatment expected to be annotated with this $\mathrm{GO}$ term under the null hypothesis; $P$-value - $P$-value of the Fisher's test found using the Weight01 algorithm of [74]; Ontology - GO category to which each GO term belongs (MF - Molecular Function; CC - Cellular Component; BP - Biological Process). (XLSX $21 \mathrm{~kb})$

\section{Abbreviations}

AMP: Antimicrobial peptide; Bt407: Bacillus thuringiensis 407; Btt: Bacillus thuringiensis bv. tenebrionis; Cro 1: Croatia 1 beetle population; Dscam: Down syndrome cell-adhesion molecule; GO: Gene ontology; IMD: Immune deficiency pathway; PCA: Principle components analysis; ROS: Reactive oxygen species

\section{Acknowledgements}

We thank Hendrik Eggert and Erich Bornberg-Bauer for helpful discussions of the data and Matthew Grigg for help with the illustrations. Furthermore, we thank the iBeetle consortium, in particular Gregor Bucher and the group of Mario Stanke for providing unpublished RNA and EST data. We would further like to thank the three anonymous reviewers for their helpful comments on the manuscript.

\section{Funding}

This work was supported by grants BO 2544/7-1, KU 1929/4-2, RO 2994/3-2, SCHU 1415/9-2 within the DFG (German Research Foundation, http://dfg.de/) priority programme 1399 "Host-parasite coevolution". The funding body had no influence on the design of the study and collection, analysis, and interpretation of data and in writing the manuscript.

\section{Availability of data and materials}

The data sets supporting the results of this article are available in the National Center for Biotechnology Information (NCBI) Sequence Read Archive (SRA), accession number SRP067120.

\section{Authors' contributions}

$\mathrm{JK}, \mathrm{BM}$ and RP conceived the study. BM, RP, SB, PR, HS, and JK participated in the design of the study. BM performed the experiments. DE and PR sequenced the transcriptome. JMG performed the statistical and bioinformatic analyses. $J M G, B M, R P$ and JK interpreted the data. JMG, BM, RP and JK drafted the paper. All authors read and approved the manuscript.

\section{Competing interests}

The authors declare that they have no competing interests.

\section{Consent for publication}

Not applicable.

\section{Ethics approval and consent to participate}

T. castaneum insects used in the study are derived from own laboratory populations. According to German law, no ethics approval is required for the work with insect species.

\section{Publisher's Note}

Springer Nature remains neutral with regard to jurisdictional claims in published maps and institutional affiliations.

\section{Author details}

'Institute for Evolution and Biodiversity, University of Münster, Hüfferstrasse 1, 48149 Münster, Germany. ${ }^{2}$ Institute of Science and Technology Austria, Am Campus 1, 3400 Klosterneuburg, Austria. ${ }^{3}$ Current Address: Stowers Institute for Medical Research, 1000 East 50th Street, Kansas City, MO 64110, USA. ${ }^{4}$ Institute of Clinical Molecular Biology, Christian-Albrechts University Kiel, Schittenhelmstr. 12, 24105 Kiel, Germany. ${ }^{5}$ Zoological Institute, Christian-Albrechts University Kiel, Am Botanischen Garten 1-9, 24118 Kiel, Germany.
Received: 24 December 2016 Accepted: 12 April 2017

Published online: 26 April 2017

\section{References}

1. Woolhouse MEJ, Webster JP, Domingo E, Charlesworth B, Levin BR. Biological and biomedical implications of the co-evolution of pathogens and their hosts. Nat Genet. 2002;32:569-77.

2. Schulenburg H, Kurtz J, Moret $Y$, Siva-Jothy MT. Introduction. Ecological immunology. Philos Trans R Soc Lond B Biol Sci. 2009;364:3-14.

3. Kurtz J, Schulenburg H, Reusch TBH. Host-parasite coevolution - rapid reciprocal adaptation and its genetic basis. Zoology. 2016;119:241-3.

4. McTaggart SJ, Wilson PJ, Little TJ. Daphnia magna shows reduced infection upon secondary exposure to a pathogen. Biol Lett. 2012:8:972-5.

5. Moret $Y$, Siva-Jothy MT. Adaptive innate immunity? Responsive-mode prophylaxis in the mealworm beetle, Tenebrio molitor. Proc Biol Sci. 2003;270:2475-80.

6. Sadd BM, Schmid-Hempel P. Insect immunity shows specificity in protection upon secondary pathogen exposure. Curr Biol. 2006;16:1206-10.

7. Kurtz J. Specific memory within innate immune systems. Trends Immunol. 2005:26:186-92.

8. Milutinović B, Kurtz J. Immune memory in invertebrates. Semin Immunol. 2016:28:328-42.

9. Roth O, Sadd BM, Schmid-Hempel P, Kurtz J. Strain-specific priming of resistance in the red flour beetle, Tribolium castaneum. Proc R Soc Biol Sci. 2009;276:145-51.

10. Kurtz J, Franz K. Evidence for memory in invertebrate immunity. Nature. 2003;425:37-8.

11. Criscitiello MF, de Figueiredo P. Fifty shades of immune defense. PLoS Pathog. 2013;9, e1003110.

12. Pham LN, Dionne MS, Shirasu-Hiza M, Schneider DS. A specific primed immune response in Drosophila is dependent on phagocytes. PLoS Pathog. 2007;3, e26.

13. Freitak D, Heckel DG, Vogel H. Dietary-dependent trans-generational immune priming in an insect herbivore. Proc Biol Sci. 2009;276:2617-24.

14. Rodrigues J, Brayner FA, Alves LC, Dixit R, Barillas-Mury C. Hemocyte differentiation mediates innate immune memory in Anopheles gambiae mosquitoes. Science. 2010;329:1353-5.

15. Milutinović B, Fritzlar S, Kurtz J. Increased survival in the red flour beetle after oral priming with bacteria-conditioned media. J Innate Immun. 2014;6:306-14

16. Milutinović B, Peuß R, Ferro K, Kurtz J. Immune priming in arthropods: an update focusing on the red flour beetle. Zoology. 2016;119:254-61.

17. Armitage $\mathrm{SAO}$, Peuß R, Kurtz J. Dscam and pancrustacean immune memory - A review of the evidence. Dev Comp Immunol. 2015;48:315-23.

18. Pascual L, Jakubowska AK, Blanca JM, Cañizares J, Ferré J, Gloeckner G, et al. The transcriptome of Spodoptera exigua larvae exposed to different types of microbes. Insect Biochem Mol Biol. 2012;42:557-70.

19. Behrens S, Peuß R, Milutinović B, Eggert H, Esser D, Rosenstiel P, et al. Infection routes matter in population-specific responses of the red flour beetle to the entomopathogen Bacillus thuringiensis. BMC Genomics. 2014;15:445.

20. Barribeau SM, Schmid-Hempel P, Sadd BM. Royal Decree: Gene expression in trans-generationally immune primed bumblebee workers mimics a primary immune response. PLoS One. 2016;11, e0159635.

21. Pinaud S, Portela J, Duval D, Nowacki FC, Olive M-A, Allienne J-F, et al. A shift from cellular to humoral responses contributes to innate immune memory in the vector snail Biomphalaria glabrata. PLoS Pathog. 2016;12, e1005361.

22. Padin S, Bello GD, Fabrizio M. Grain loss caused by Tribolium castaneum, Sitophilus oryzae and Acanthoscelides obtectus in stored durum wheat and beans treated with Beauveria bassiana. I Stored Prod Res. 2002;38:69-74.

23. Richards S, Gibbs RA, Weinstock GM, Brown SJ, Denell R, Beeman RW, et al. The genome of the model beetle and pest Tribolium castaneum. Nature. 2008;452:949-55.

24. Milutinović B, Stolpe C, Peuß R, Armitage SAO, Kurtz J. The red flour beetle as a model for bacterial oral infections. PLoS One. 2013;8:e64638.

25. Eggert H, Diddens-de Buhr MF, Kurtz J. A temperature shock can lead to trans-generational immune priming in the red flour beetle, Tribolium castaneum. Ecol Evol. 2015;5:1318-26. 
26. Zou Z, Evans JD, Lu Z, Zhao P, Williams M, Sumathipala N, et al. Comparative genomic analysis of the Tribolium immune system. Genome Biol. 2007:8:R177.

27. Gillespie JP, Kanost MR, Trenczek T. Biological mediators of insect immunity. Annu Rev Entomol. 1997;42:611-43.

28. Adamo SA. Estimating disease resistance in insects: phenoloxidase and lysozyme-like activity and disease resistance in the cricket Gryllus texensis. J Insect Physiol. 2004;50:209-16.

29. Van Herreweghe JM, Michiels CW. Invertebrate lysozymes: Diversity and distribution, molecular mechanism and in vivo function. J Biosci. 2012;37: 327-48.

30. Zelensky AN, Gready JE. The C-type lectin-like domain superfamily. FEBS J. 2005;272:6179-217.

31. Schnitger AKD, Yassine H, Kafatos FC, Osta MA. Two c-type lectins Cooperate to defend Anopheles gambiae against gram-negative bacteria. J Biol Chem. 2009;284:17616-24.

32. Shi X-Z, Kang C-J, Wang S-J, Zhong X, Beerntsen BT, Yu X-Q. Functions of Armigeres subalbatus c-type lectins in innate immunity. Insect Biochem Mol Biol. 2014;52:102-14.

33. Pees B, Yang W, Zárate-Potes A, Schulenburg H, Dierking K. High innate immune specificity through diversified c-type lectin-like domain proteins in invertebrates. J Innate Immun. 2016:8:129-42.

34. Shrestha S, Kim Y. An entomopathogenic bacterium, Xenorhabdus nematophila, inhibits hemocyte phagocytosis of Spodoptera exigua by inhibiting phospholipase A2. J Invertebr Pathol. 2007;96:64-70.

35. Shrestha S, Kim Y. Activation of immune-associated phospholipase A2 is functionally linked to Toll/Imd signal pathways in the red flour beetle, Tribolium castaneum. Dev Comp Immunol. 2010;34:530-7

36. Kim Y, Ji D, Cho S, Park Y. Two groups of entomopathogenic bacteria, Photorhabdus and Xenorhabdus, share an inhibitory action against phospholipase A2 to induce host immunodepression. J Invertebr Pathol. 2005:89:258-64.

37. Miller JS, Nguyen T, Stanley-Samuelson DW. Eicosanoids mediate insect nodulation responses to bacterial infections. Proc Natl Acad Sci U S A. 1994; 91:12418-22.

38. Freitak D, Wheat CW, Heckel DG, Vogel H. Immune system responses and fitness costs associated with consumption of bacteria in larvae of Trichoplusia ni. BMC Biol. 2007:5:56.

39. Contreras E, Rausell C, Real MD. Proteome response of Tribolium castaneum larvae to Bacillus thuringiensis toxin producing strains. PLoS One. 2013;8, e55330

40. Fallon JP, Troy N, Kavanagh K. Pre-exposure of Galleria mellonella larvae to different doses of Aspergillus fumigatus conidia causes differential activation of cellular and humoral immune responses. Virulence. 2011;2: 413-21.

41. Zhu J-Y, Yang $P$, Zhang Z, Wu G-X, Yang B. Transcriptomic immune response of Tenebrio molitor pupae to parasitization by Scleroderma guani. PLoS One. 2013;8, e54411.

42. Wang Z, Wilhelmsson C, Hyrs I P, Loof TG, Dobes P, Klupp M, et al. Pathogen entrapment by transglutaminase-A conserved early innate immune mechanism. PLoS Pathog. 2010;6, e1000763.

43. Ma G, Roberts H, Sarjan M, Featherstone N, Lahnstein J, Akhurst R, et al. Is the mature endotoxin Cry1Ac from Bacillus thuringiensis inactivated by a coagulation reaction in the gut lumen of resistant Helicoverpa armigera larvae? Insect Biochem Mol Biol. 2005:35:729-39.

44. Ma G, Rahman MM, Grant W, Schmidt O, Asgari S. Insect tolerance to the crystal toxins Cry1Ac and Cry2Ab is mediated by the binding of monomeric toxin to lipophorin glycolipids causing oligomerization and sequestration reactions. Dev Comp Immunol. 2012;37:184-92.

45. Mahbubur Rahman M, Ma G, Roberts HLS, Schmidt O. Cell-free immune reactions in insects. J Insect Physiol. 2006;52:754-62.

46. Qiao C, Li J, Wei X-H, Wang J-L, Wang Y-F, Liu X-S. SRP gene is required for Helicoverpa armigera prophenoloxidase activation and nodulation response. Dev Comp Immunol. 2014:44:94-9.

47. Guo Z, Cheng Zhu Y, Huang F, Luttrell R, Leonard R. Microarray analysis of global gene regulation in the Cry1Ab-resistant and Cry1Ab-susceptible strains of Diatraea saccharalis. Pest Manag Sci. 2012;68:718-30.

48. Bel Y, Jakubowska AK, Costa J, Herrero S, Escriche B. Comprehensive analysis of gene expression profiles of the beet armyworm Spodoptera exigua larvae challenged with Bacillus thuringiensis Vip3Aa toxin. PLoS One. 2013;8:1-23.
49. Schmid-Hempel P. Evolutionary Parasitology: The Integrated Study of Infections, Immunology, Ecology, and Genetics. 1st ed. Oxford: Oxford University Press; 2011.

50. Johnston PR, Makarova O, Rolff J. Inducible Defenses Stay Up Late: Temporal patterns of immune gene expression in Tenebrio molitor. G3. 2014;4:947-55.

51. Contreras-Garduño J, Lanz-Mendoza H, Franco B, Nava A, Pedraza-Reyes M, Canales-Lazcano J. Insect immune priming: ecology and experimental evidences. Ecol Entomol. 2016;41:351-66.

52. Coustau C, Kurtz J, Moret Y. A novel mechanism of immune memory unveiled at the invertebrate-parasite interface. Trends Parasitol. 2016;32:353-5.

53. Yokoi K, Koyama H, Minakuchi C, Tanaka T, Miura K. Antimicrobial peptide gene induction, involvement of Toll and IMD pathways and defense against bacteria in the red flour beetle, Tribolium castaneum. Results Immunol. 2012;2:72-82.

54. Koyama H, Kato D, Minakuchi C, Tanaka T, Yokoi K, Miura K. Peptidoglycan recognition protein genes and their roles in the innate immune pathways of the red flour beetle, Tribolium castaneum. J Invertebr Pathol. 2015;132:86-100.

55. Shah N, Dorer DR, Moriyama EN, Christensen AC. Evolution of a large, conserved, and syntenic gene family in insects. G3. 2012;2:313-9.

56. Vilcinskas A, Vogel H. Seasonal phenotype-specific transcriptional reprogramming during metamorphosis in the European map butterfly Araschnia levana. Ecol Evol. 2016;6:3476-85.

57. Matzinger $P$. The danger model: a renewed sense of self. Science. 2002;296:301-5.

58. Ramirez JL, de Almeida Oliveira G, Calvo E, Dalli J, Colas RA, Serhan CN, et al. A mosquito lipoxin/lipocalin complex mediates innate immune priming in Anopheles gambiae. Nat Commun. 2015:6:7403.

59. Futo M, Armitage SAO, Kurtz J. Microbiota plays a role in oral immune priming in Tribolium castaneum. Front Microbiol. 2016;6:1383.

60. Sheldon BC, Verhulst S. Ecological immunology. TREE. 1996;11(8):317-21.

61. Milutinović B, Höfling C, Futo M, Scharsack JP, Kurtz J. Infection of Tribolium castaneum with Bacillus thuringiensis: quantification of bacterial replication within cadavers, transmission via cannibalism, and inhibition of spore germination. Appl Environ Microbiol. 2015;81:8135-44.

62. St John J. SeqPrep. https://github.com/jstjohn/SeqPrep. Accessed Sept 2013.

63. Li H. Seqtk. https://github.com//h3/seqtk. Accessed Sept 2013.

64. Hansen KD, Brenner SE, Dudoit S. Biases in Illumina transcriptome sequencing caused by random hexamer priming. Nucleic Acids Res. 2010;38:e131.

65. Trapnell C, Pachter L, Salzberg SL. TopHat: discovering splice junctions with RNA-Seq. Bioinformatics. 2009;25:1105-11.

66. Kim HS, Murphy T, Xia J, Caragea D, Park Y, Beeman RW, et al. BeetleBase in 2010: revisions to provide comprehensive genomic information for Tribolium castaneum. Nucleic Acids Res. 2010;38:D437-42.

67. Donitz J, Schmitt-Engel C, Grossmann D, Gerischer L, Tech M, Schoppmeier $M$, et al. iBeetle-Base: a database for RNAi phenotypes in the red flour beetle Tribolium castaneum. Nucleic Acids Res. 2015;43: D720-5.

68. Schmitt-Engel C, Schultheis D, Schwirz J, Ströhlein N, Troelenberg N Majumdar $U$, et al. The iBeetle large-scale RNAi screen reveals gene functions for insect development and physiology. Nat Commun. 2015;6: 7822 .

69. Trapnell C, Williams BA, Pertea G, Mortazavi A, Kwan G, van Baren MJ, et al, Transcript assembly and quantification by RNA-Seq reveals unannotated transcripts and isoform switching during cell differentiation. Nat Biotechnol. 2010;28:511-5.

70. Trapnell C, Hendrickson DG, Sauvageau M, Goff L, Rinn JL, Pachter L. Differential analysis of gene regulation at transcript resolution with RNA-seq Nat Biotechnol. 2013;31:46-53.

71. R Core Team. R: A language and environment for statistical computing. Vienna: R Foundation for Statistical Computing; 2013.

72. Chen $H$, Boutros PC. VennDiagram: a package for the generation of highlycustomizable Venn and Euler diagrams in R. BMC Bioinformatics. 2011;12:35.

73. Conesa A, Götz S, García-Gómez JM, Terol J, Talón M, Robles M. Blast2GO: a universal tool for annotation, visualization and analysis in functional genomics research. Bioinformatics. 2005:21:3674-6.

74. Alexa A, Lengauer T. Gene expression Improved scoring of functional groups from gene expression data by decorrelating $\mathrm{GO}$ graph structure. Bioinformatics. 2006;22:1600-7.

75. Moore AD, Bornberg-Bauer E. The dynamics and evolutionary potential of domain loss and emergence. Mol Biol Evol. 2012;29:787-96. 
76. The Gene Ontology Consortium. The Gene Ontology's Reference Genome Project: a unified framework for functional annotation across species. PLoS Comput Biol. 2009;5:1000431.

77. Smedley D, Haider S, Durinck S, Pandini L, Provero P, Allen J, et al. The BioMart community portal: an innovative alternative to large, centralized data repositories. Nucleic Acids Res. 2015;43:589-98.

78. Altschul SF, Gish W, Miller W, Myers EW, Lipman DJ. Basic local alignment search tool. J Mol Biol. 1990;215:403-10.

79. Benjamini Y, Hochberg Y. Controlling the false discovery rate - a practical and powerful approach to multiple testing. J R Stat Soc Ser B-Methodological. 1995:57:289-300

Submit your next manuscript to BioMed Central and we will help you at every step:

- We accept pre-submission inquiries

- Our selector tool helps you to find the most relevant journal

- We provide round the clock customer support

- Convenient online submission

- Thorough peer review

- Inclusion in PubMed and all major indexing services

- Maximum visibility for your research

Submit your manuscript at www.biomedcentral.com/submit 